\title{
Fish consumption and polyunsaturated fatty acid status of pregnant women in the Seychelles Child Development and Nutrition Study Cohort 2
}

\author{
A. J. Yeates ${ }^{1}$, M. S. Mulhern ${ }^{1}$, E. M. McSorley ${ }^{1}$, J. M. W. Wallace ${ }^{1}$, G. E. Watson ${ }^{2}$, G. J. Myers ${ }^{2}$, E. \\ van Wijngaarden ${ }^{2}$, C. Shamlaye ${ }^{3}$, J. Henderson ${ }^{3}$, S. W. Thurston ${ }^{2}$, P. W. Davidson ${ }^{2}$ and J. J. Strain ${ }^{1}$ \\ ${ }^{1}$ Northern Ireland Centre for Food and Health, University of Ulster, Coleraine, BT52 1SA, ${ }^{2}$ University of Rochester, \\ School of Medicine and Dentistry, NY, USA and ${ }^{3}$ Ministry of Health, Victoria, Mahé, Republic of Seychelles
}

Fish is the major dietary source of long chain polyunsaturated fatty acids (PUFA), including docosahexaenoic acid (DHA), which is essential for optimal child neurodevelopment ${ }^{(1)}$. In the first nutrition cohort of the Seychelles Child Development Study (SCDS), we showed no significant relationship between fish consumption (average 12 meals per week) and DHA status of pregnant women ${ }^{(2)}$. We hypothesised that altered fatty acid metabolism during pregnancy could be obscuring any correlation between dietary intake data and physiological measures. In the present study, we repeated the analysis to look at associations between fish consumption and PUFA status in the larger second nutrition SCDS cohort.

Fish consumption was assessed as frequency of fish meals consumed per week using a fish use questionnaire (FUQ) administered during pregnancy. Lipid extractions were carried out on serum samples collected at 28 weeks gestation following the Folch et al. method ${ }^{(3)}$ and fatty acid methyl esters were determined by GC-MS, as previously described ${ }^{(2)}$. A total of $n=1474$ women completed the FUQ and provided a blood sample for PUFA measurement. Descriptive statistics and Pearson correlation analysis was carried out using IBM SPSS Statistics $v .20$.

\begin{tabular}{lccc}
\hline & Mean & SD & $5^{\text {th }}, 95^{\text {th }}$ centile \\
\hline Fish meals per week & 8.59 & 4.58 & $3.00,17.00$ \\
$n$-6 PUFA $m g / m l$ & & & \\
LA & 0.902 & 0.255 & $0.526,1.340$ \\
AA & 0.203 & 0.082 & $0.061,0.334$ \\
Total $n-6$ & 1.105 & 0.295 & $0.659,1.608$ \\
$n-3$ PUFA $m g / m l$ & & & \\
ALA & 0.037 & 0.006 & $0.035,0.047$ \\
EPA & 0.052 & 0.008 & $0.049,0.069$ \\
DHA & 0.185 & 0.086 & $0.049,0.329$ \\
Total $n$-3 & 0.274 & 0.091 & $0.147,0.438$ \\
$n-6: n-3$ ratio & $4.358^{*}$ & 1.636 & $2.506,7.697$ \\
\hline * Values were significantly correlated with fish meals per week in Pearson correlation \\
$\quad(P<0.05)$
\end{tabular}

The mean age of pregnant women at enrolment to the study was $26(\mathrm{SD}=6)$ years and the mean number of fish meals consumed per week during pregnancy was reported as $8.59(\mathrm{SD}=4.58)$. Fish consumption was not significantly correlated with either individual or summed concentrations of $n-3$ or $n-6$ PUFA; however it was significantly negatively associated with the ratio of $n-6: n-3$ ( $r=-0.06$, $P=0.04)$.

These results agree with those of a previous SCDS cohort, where fish consumption, albeit assessed by food diary and in a smaller cohort $(n \text { 229) })^{(2)}$, was not significantly associated with serum PUFA status in pregnant women. This finding highlights a potential limitation of using fish consumption data as a biomarker for PUFA status, particularly during pregnancy. Further studies to identify a more robust biomarker for fish consumption are warranted.

1. Carlson SE \& Neuringer (1999) Lipids 34, 171-178.

2. Bonham MP, Duffy EM, Wallace JMW et al. (2008) Prostag Leukotr Ess 78, 343-350.

3. Folch J, Lees M \& Stanley GH (1957) J Biol Chem 226, 497-509. 